\title{
Weisberg on design: What fine-tuning's got to do with it
}

(Penultimate draft of Erkenntnis 77 (3):435-438 (2012))

Jonathan Weisberg (2010) argues that, given the old datum that life exists, the new datum that the universe is fine-tuned for life does not confirm the design hypothesis. Thus, finetuning has nothing to do with it. I will argue that there is a design argument that survives Weisberg's critique; the fact that the universe is life-sustaining supports the design hypothesis, but it only does so given fine-tuning.

\section{The Fine-Tuning Argument}

It is a striking fact that the universe appears fine-tuned for life. That is, if the constants of the laws of nature had been even slightly different, the result would have been a universe that could not sustain life. ${ }^{1}$ This fact can be used to support the hypothesis that the universe was created by an intelligent designer. Here is how Weisberg puts the argument he goes on to reject:

$[F]$ ine-tuning is to be expected if the universe was created by a designer of the sort typically postulated in western monotheistic traditions. Such a designer wants to create intelligent life, and can thus be expected to set the relevant values so as to allow for intelligent life. But if the universe was not created by such a designer, there is no reason to expect these values to be as we find them. Indeed, it seems much more probable that the actual values would fall outside of the small range necessary for intelligent life.

\footnotetext{
${ }^{1}$ See for example Collins, Rees and McMullin.
} 
So the fact that fine-tuning obtains favours the design hypothesis over its negation. p.432

We can put the argument more formally using the following abbreviations and the Law of Likelihood (Hacking 1965):

$\mathrm{N}=$ Only a narrow range of constants sustain life / Fine-tuning ${ }^{2}$

$\mathrm{D}=$ There is an intelligent designer

Law of likelihood: E supports H1 over H2 given background beliefs B iff $\mathrm{P}(\mathrm{E} \mid \mathrm{H} 1 \& \mathrm{~B})>\mathrm{P}(\mathrm{E} \mid-\mathrm{H} 2 \& \mathrm{~B})$

The fine-tuning argument uses the premise that $\mathrm{P}(\mathrm{N} \mid \mathrm{D})>\mathrm{P}(\mathrm{N} \mid-\mathrm{D})$ and concludes that $\mathrm{N}$ supports D over - D. ${ }^{3}$

\section{Weisberg's Objection}

Weisberg objects that we have no reason to believe that $\mathrm{P}(\mathrm{N} \mid \mathrm{D})>\mathrm{P}(\mathrm{N} \mid-\mathrm{D})$. His argument can be summed up with one rhetorical question: 'Given the old datum that intelligent life exists... why would fine-tuning be any more probable if there were a designer than if there weren't?' p. 435

\footnotetext{
${ }^{2}$ The expression 'fine-tuning' is naturally understood as the conjunction of 'only a narrow range of constants sustains life' and 'the universe has constants that sustain life'. These are the New Datum and the Old Datum that Weisberg correctly points out we need to keep separate. Conflating them adds to the plausibility of the first sentence of the quote from the Weisberg paper. I follow Weisberg in using 'finetuning' to mean 'only a narrow range of constants supports life'.

${ }^{3}$ I'll leave 'over -D' implicit in future.
} 
Weisberg argues that it would not be. A designer could have created a universe in which a broad range of constants sustained life, or a universe in which only a narrow range of constants sustains life. We have no reason to think one more likely than the other. Formally, $\mathrm{P}(\mathrm{N} \mid \mathrm{D})>\mathrm{P}(\mathrm{N} \mid-\mathrm{D})$ says that a designer is more likely than a mindless process to create a universe in which only a narrow range of constants sustains life. But we have no reason to believe this. So we have no reason to accept the first sentence of the quotation above. I will grant Weisberg this point for the sake of argument. ${ }^{4}$

Weisberg leaves it open whether a different fact supports D - namely the fact that the universe does have the right constants for life. Call this L. But if it is L that supports D, fine-tuning seems to have nothing to do with it! And this is puzzling, as it is the apparent degree of fine-tuning that has generated the recent interest in the argument. ${ }^{5}$ So have all those who thought that fine-tuning confirmed design just been confused?

\section{Response}

I don't think so. I argue that L does support D, but it does so only on the condition that a narrow range of constants sustains life - and the narrower the range, the greater the confirmation. That's what fine-tuning has to do with it.

To take the extreme case first, suppose that any constants would sustain life. (Call this $\mathrm{B}$ for Broad $)$. Then $\mathrm{P}(\mathrm{L} \mid-\mathrm{D} \& \mathrm{~B})=1$. Assuming that a designer would have ensured life existed, $\mathrm{P}(\mathrm{L} \mid \mathrm{D} \& \mathrm{~B})=1$. So $\mathrm{P}(\mathrm{L} \mid \mathrm{D} \& \mathrm{~B})=\mathrm{P}(\mathrm{L} \mid-\mathrm{D} \& \mathrm{~B})$, and $\mathrm{L}$ would not confirm $\mathrm{D}$.

\footnotetext{
${ }^{4}$ White (forthcoming) convincingly responds to Weisberg's argument here. I argue that even if we grant Weisberg this point, there is still a fine-tuning argument which takes $\mathrm{L}$ as the new evidence.

${ }^{5}$ There is no reason to think this objection limited to the cosmological fine-tuning argument as opposed to the traditional biological design argument. Weisberg doesn't extend his objection in this way, but I see no reason why he shouldn't.
} 
Now suppose that only a narrow range of constants would generate life $(\mathrm{N})$. If there is no designer we would expect the probability of $\mathrm{L}$ to be less than $1 ; \mathrm{P}(\mathrm{L} \mid-\mathrm{D} \& \mathrm{~N})<$ 1. Again assuming that a designer would have ensured life existed, $P(L \mid D \& N)=1$. So $\mathrm{P}(\mathrm{L} \mid-\mathrm{D} \& \mathrm{~N})<\mathrm{P}(\mathrm{L} \mid \mathrm{D} \& \mathrm{~N})$, and L supports D. So L supports $\mathrm{D}$ only if it is not part of the background condition that all constants generate life. So some degree of fine-tuning is essential to the confirmation of D after all.

\section{Generalizing the Response}

The point can be generalized. Let's assume that we can model the values of constants as being fixed by a random process if there is no designer. Then we can show that the narrower the range of constants that allow life, the greater the degree of confirmation $\mathrm{L}$ gives D.

Let $\mathrm{C}_{\mathrm{x}}=$ The fraction of settings on which the constants are capable of supporting life is $\mathrm{x}$.

Thus $\mathrm{P}\left(\mathrm{L} \mid-\mathrm{D} \& \mathrm{C}_{\mathrm{x}}\right)=\mathrm{x}$

L supports $D \& C_{x}$ iff $P\left(L \mid-D \& C_{x}\right)<P\left(L \mid D \& C_{x}\right)$, and the degree of confirmation increases with the size of the inequality. The size of the inequality is maximal if $\mathrm{P}(\mathrm{L} \mid \mathrm{D} \&$ $\left.C_{x}\right)=1$ (i.e. a designer would ensure life exists), and $P\left(L \mid-D \& C_{x}\right)=x$ is vanishingly

small. As $x \rightarrow 1$, and the range of life-supporting constants increases, the size of the inequality decreases. So the smaller the value of $\mathrm{x}$, the narrower the life-supporting range, and the greater the degree of confirmation $\mathrm{L}$ gives $\mathrm{D} \& \mathrm{C}_{\mathrm{x}}$.

The assumption that an intelligent designer is guaranteed to create life can be weakened. $\mathrm{P}\left(\mathrm{L} \mid-\mathrm{D} \& \mathrm{C}_{\mathrm{x}}\right)<\mathrm{P}\left(\mathrm{L} \mid \mathrm{D} \& \mathrm{C}_{\mathrm{x}}\right)$ merely requires that the probability that a designer 
would generate life is higher than the probability that a mindless universe would. And this is surely the intuition at the heart of the design argument.

This is not to say that the fine-tuning argument is succesful, ${ }^{6}$ but I have argued that it can survive Weisberg's objection.

\section{Acknowledgements}

I am grateful to Jonthan Weisberg and two anonymous referees for this journal for helpful comments on an earlier draft.

\section{References}

Bradley, D. J. (2009) "Multiple Universes and Observation Selection Effects", American Philosophical Quarterly 46:1, 61-72.

Collins, R. (2003) 'Evidence for fine-tuning'. In God and Design: The Teleological Argument and Modern Science, ed. N. Manson. Routledge.

Hacking, I. (1965): The Logic of Statistical Inference. Cambridge: Cambridge University Press.

McMullin, E. (1993) 'Indifference Principle and Anthropic Principle in Cosmology', Studies in the History and Philosophy of Science, 24: 359-89.

Rees, M. (1999) ‘Just Six Numbers’ Basic Books.

Weisberg, J. (2010) 'A note on design: What's fine-tuning got to do with it?' Analysis $70: 3,431-438$

White (forthcoming) 'What Fine-Tuning's Got to Do With It: A Reply to Weisberg' Analysis

\footnotetext{
${ }^{6}$ My preferred response to the fine-tuning argument is that there are multiple universes (Bradley 2009).
} 